\title{
Intracranial Angiomatous Meningioma: A Clinicopathological Study of 23 Cases
}

This article was published in the following Dove Press journal:

International Journal of General Medicine

\section{Liusong Yang $\mathbb{D}^{\prime}$ \\ Guoqiang Ren ${ }^{2}$ \\ Jianmin Tang ${ }^{2}$}

'Department of Neurosurgery, Huashan Hospital North, Fudan University, Shanghai 201907, People's Republic of China; ${ }^{2}$ Department of Pathology, Huashan Hospital North, Fudan University, Shanghai 201907, People's Republic of China
Correspondence: Liusong Yang Tel +862166895203

Email yangls72@hotmail.com
Background: Intracranial angiomatous meningioma (AM) is a rare subtype of meningioma. Here, we investigated the clinical and pathological features of AMs.

Materials and Methods: We performed a retrospective study of 23 intracranial AMs verified by postoperative pathology at Huashan Hospital North between 2013 and 2018 . Clinical data, radiological and pathological findings, and information on treatment and outcomes were collected and analyzed. Additionally, the literature on intracranial AMs was reviewed.

Results: The sample comprised 13 men and 10 women with AMs. The mean age was 54.2 years, and the mean duration of symptoms was 14.9 months. Headache and epilepsy were the most common symptoms. The most common AMs locations were the cerebral convexity and parasagittal/falx region. The rates of vascular signs, homogeneous enhancement, and peritumoral brain edema (PTBE) on magnetic resonance images were high. Histologically, besides typical meningioma cells, AMs had an abundant vascular component and low Ki-67 index. The extent of PTBE was related to microvessel density (MVD) of tumors, but not to the expression of MMP9 or VEGF. Simpson grade I resection was achieved in 15 cases, and grade II resection was achieved in 7 cases. Twenty-one cases were followed up, and they all had favorable outcomes without recurrence.

Conclusion: AM is a type of meningioma with a rich blood supply and distinct clinical and pathological features. It showed a slight male predominance and was common at the cerebral convexity or parasagittal/falx region. Histologically, it showed benign biological characteristics despite frequent and severe PTBE, and the extent of PTBE was related to MVD of tumors. Simpson I resection is the best treatment, and the prognosis is usually good after total tumor removal, while gamma knife is recommended for small residual tumor.

Keywords: angiomatous meningioma, clinical characteristic, radiology, pathohistology, prognosis

\section{Introduction}

Angiomatous meningioma (AM) is a rare subtype of meningioma, which accounts for $2.1-2.59 \%$ of all meningiomas. ${ }^{1,2}$ Due to its extremely low incidence, most neurosurgeons are not sufficiently familiar with its clinical features. The rate of misdiagnosis before surgery is high, which results in increased intraoperative bleeding, leading to the impairment of neurological function.

Because of its extremely rich blood supply, surgical resection of an AM is more difficult than resection of other types of meningiomas. Frequent and severe peritumoral brain edema (PTBE) is an important characteristic of AM. However, the possible molecular mechanism is not clear. 
This retrospective analysis of 23 cases of intracranial AM in patients admitted to our department between 2013 and 2018 was conducted to further explore the clinical and pathological characteristics of AM.

\section{Materials and Methods}

From January 2013 to December 2018, 23 patients with surgically confirmed AM were admitted to Huashan Hospital North. Clinical data including age, sex, clinical symptoms, histopathology findings, radiologic manifestations, tumor characteristics, and extent of surgical resection were collected from electronic medical records of all patients. Informed patient consent was obtained from all of the subjects, and the study was conducted in compliance with the approval from the review board of Huashan Hospital North, Fudan University for research on human subjects.

All of the patients underwent cranial computed tomography (CT) and magnetic resonance imaging (MRI) (unenhanced and enhanced) examinations before surgery. Magnetic resonance venogram (MRV) examinations were performed for parasagittal tumors. CT angiography (CTA) or magnetic resonance angiography (MRA) were conducted for skull base tumors. Digital subtraction angiography (DSA) was performed if clinicians considered that embolization was necessary for the treatment of the tumor. The average tumor diameter was based on the maximum diameter of tumor enhancement on MRI. According to the standard of Liu et al, the width of peritumoral brain edema at the largest tumor level on T2-weighted magnetic resonance images was classified as mild $(\leq 2 \mathrm{~cm})$, moderate $(>2 \mathrm{~cm}$, and $\leq 1 / 2$ hemisphere), and severe $(>1 / 2$ hemisphere). ${ }^{3}$ All of the cases were treated surgically, and the extent of tumor resection was judged by the enhanced MRI three months later in combination with surgical records in accordance with the Simpson grading criteria. ${ }^{4}$

All of the specimens were sent for postoperative pathological examination. Tumor specimens were fixed in $10 \%$ formalin, embedded in paraffin, and $4 \mathrm{~mm}$ thick sections were stained with hematoxylin and eosin. The sections for immunohistochemistry (IHC) were stained for EMA (1:200; Long Island Antibody Diagnostic Company, Shanghai, China), vimentin (1:200; Shenda Bio, Guangzhou, China), CD34 (1:100; Long Island Antibody Diagnostics Company), Ki-67 (1:200; Shenda Bio), VEGF (1:100; Maxim Biotechnologies Company, Fuzhou, China), and MMP9 (1:100; Abcam Company, Cambridge, England). The IHC staining was evaluated in 10 random fields under $20 \times$ magnification by two independent pathologists (Tang and Zhu).
As the previous report of Hasselblatt et al, a total score, which was the sum of the scores of immunopositivity $(0$, $0 \% ; 1,<10 \% ; 2,10 \%-50 \%, 3,51 \%-80 \%$; and $4,81-100 \%$ ) and staining intensity $(0$, none; 1 , weak; 2 , moderate; and 3 , intense), was considered positive if it was $\geq 2 .{ }^{1} \mathrm{Ki}-67$ index was estimated as the percentage of $\mathrm{Ki}-67$ positive tumor cells. According to the modified method of Weidner et al, ${ }^{5}$ microvessel density (MVD) was counted as microvessels stained for CD34 per 400× field in the three most active areas of neovascularization chosen at a low-power $(100 \times)$ field. The relationships between the extent of PTBE in AMs (divided into severe and non-severe groups) and MVD and the IHC scores of VEGF and MMP9 were evaluated by unpaired $t$-tests (GraphPad Prism 6.0).

Enhanced MRI examinations were performed 3 months after surgery in all of the patients, and then every 6 months for the first 2 years, and once a year thereafter. The followup was conducted mainly through outpatient visits and telephone communication.

\section{Results Clinical Presentation}

The sample comprised 13 men and 10 women (male/ female ratio, 1.3), with a mean age of 54.2 years (range, 34-74 years). The mean duration of symptoms was 14.9 months (range, 10 days-10 years). The most common symptoms and signs were headache and epilepsy $(n=8$ each), followed by limb weakness or paresthesia $(n=4)$, speech disorder $(n=3)$, dizziness $(n=3)$, cognitive impairment $(n=2)$, and blurred vision $(n=1)$. Three patients showed no symptoms on admission; the tumors were detected incidentally on a routine CT or MRI examination. One was a recurrence case of the tumor resected 7 years ago at another hospital. The tumors were located in the parasagittal region/cerebral falx $(n=11)$, at the cerebral convexity $(n=9)$, and at the skull base ( $n=3$; two at the sphenoidal ridge, and one in the parasellar region).

\section{Radiological Findings}

Preoperative imaging characteristics are summarized in Table 1, and representative magnetic resonance images are provided in Figure 1. The average tumor diameter was $4.0 \mathrm{~cm}$. Tumor calcification and hemorrhage were present in one case $(4.35 \%)$ each. Cystic changes were observed in six $(26.09 \%)$ tumors. Signal hypointensity on T1-weighted sequences was observed in $16(69.57 \%)$ cases, and signal hyperintensity on T2-weighted sequences 
Table I Radiological Characteristics

\begin{tabular}{|c|c|}
\hline Tumor Diameter & $4.0 \mathrm{~cm}$ (Range: $2 \mathrm{~cm}-7 \mathrm{~cm}$ ) \\
\hline Cystic change & $6(26.09 \%)$ \\
\hline Calcification & I (4.35\%) \\
\hline Tumor hemorrhage & I (4.35\%) \\
\hline \multicolumn{2}{|l|}{ MRI TI } \\
\hline Hyposignal & $16(69.57 \%)$ \\
\hline Isosignal & $7(30.43 \%)$ \\
\hline Hypersignal & 0 \\
\hline \multicolumn{2}{|l|}{ MRI T2 } \\
\hline Hyposignal & 0 \\
\hline Isosignal & $5(22.74 \%)$ \\
\hline Hypersignal & 18 (78.26\%) \\
\hline \multicolumn{2}{|l|}{ Enhancement } \\
\hline Homogeneous & 15 (65.22\%) \\
\hline Heterogeneous & $8(34.78 \%)$ \\
\hline \multicolumn{2}{|l|}{ Peritumor brain edema* } \\
\hline No & $3(13.04 \%)$ \\
\hline Mild & 6 (26.09\%) \\
\hline Moderate & $2(8.70 \%)$ \\
\hline Severe & $12(52.17 \%)$ \\
\hline Vascular signal voids & $20(86.96 \%)$ \\
\hline Dural tail sign & II (47.83\%) \\
\hline Clear arachnoid boundary & 14 (60.87\%) \\
\hline
\end{tabular}

was observed in $18(78.26 \%)$ cases. PTBE and vascular signal voids were seen in 20 cases $(86.96 \%)$ each. The dural tail sign was observed in $11(47.83 \%)$ cases. Clear arachnoid boundaries were apparent in $14(60.87 \%)$ cases.

\section{Treatment results}

Simpson grade I resection was achieved in 15 cases, grade II resection was achieved in 7 cases, and a subtotal removal of a parasellar tumor was achieved due to invasion of the cavernous sinus. Intraoperatively, all tumors showed a rich blood supply. For tumors at the cerebral convexity, en bloc resection was adopted to achieve Simpson grade I resection. For parasagittal/falx region tumors, en bloc resection was performed as much as possible after resection of the tumor bases to achieve Simpson grade I or II resection. For skull base tumors, piecemeal tumor removal and resection of the tumor bases were suitable to avoid damaging the surrounding crucial anatomical structures; thus, Simpson grade II resection was the goal for most AMs of the skull base. One left frontal, parasagittal AM in our series was a recurrence case, diagnosed 7 years after the initial Simpson grade II resection at another hospital; we ligated the sagittal sinus and performed Simpson grade
I resection because the tumor was located in the anterior third of the anterior sagittal sinus. Twenty patients lost little blood and required no transfusion, and the other three patients were transfused with 1600,800 , and $400 \mathrm{~mL}$ of blood, respectively. These three cases comprised a giant sphenoid crest tumor and two giant parasagittal/falx tumors. Most patients showed improved neurological function, and no new neurological functional damage occurred after surgery. One patient with a tumor in the region affecting limb function showed postoperative limb muscle weakness. Another patient developed meningitis after surgery, which was treated successfully by antibiotics and lumbar drainage. Secondary epilepsy occurred after surgery in one case, and the patient's status improved after antiepileptic drug treatment. The subtotally removed parasellar tumor was treated with a gamma knife procedure 3 months after the initial surgery; other patients did not undergo postoperative radiation therapy.

\section{Pathological Findings}

Histologically, all of the tumors were composed of meningothelial and vascular elements, and the latter occupied $>50 \%$ of the total tumor area. IHC analysis showed positive expression of EMA and vimentin in all cases, positive expression of VEGF in 20 cases, and positive expression of MMP9 in 15 cases. Ki-67 indexes ranged from $0 \%$ to $5 \%$, with an average of $2.22 \%$. Among the IHC scores of VEGF and MMP9 and MVD marked with CD34 (Figure 2), only MVD correlated significantly with the extent of PTBE extent $(\mathrm{P}<0.01)$ (Figure 3$)$. The patients with or without severe PTBE have $61.08 \pm 6.351$ and 38.75 \pm 4.187 microvessels per $400 \times$ field, respectively.

\section{Follow-Up}

Follow-up data were available for 21 cases; two cases were lost to follow-up. The duration of the follow-up was 19-77 months, with a mean of 51.95 months. All of the patients were alive and in good condition at the last follow-up visit. One patient with postoperative hemiplegia recovered muscle strength almost to the preoperative state. The most recent enhanced MRI examinations showed no signs of tumor recurrence in any of the cases. The patient with subtotal tumor resection and subsequent gamma knife therapy also showed no tumor growth on the latest MRI images.

\section{Discussion}

$\mathrm{AM}$ is one of the 15 subtypes of meningioma. Its most distinctive characteristic is that the tumor is rich in vascular 

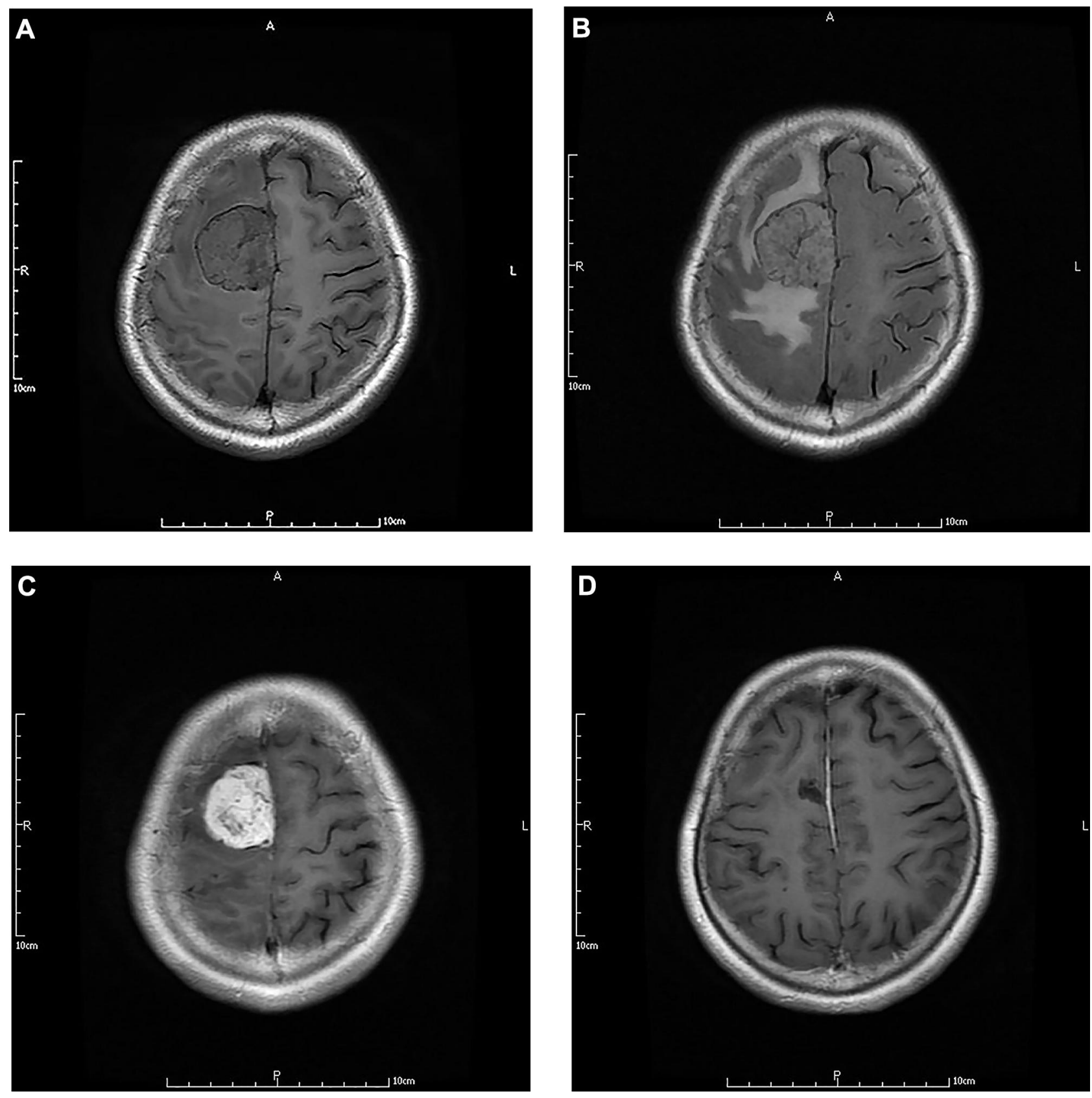

Figure I MRI of a 5 I-year-old female with AM in the right frontal parafalx region. (A) TI-weighted image showing slight signal hypointensity, a clear arachnoid interface, and obvious vascular signal voids. (B) T2-weighted image showing slight signal hyperintensity, vascular signal voids, and pronounced peritumoral brain edema. (C) Gd-enhanced image showing homogeneous enhancement. (D) Gd-enhanced image showing total absence of the tumor at three months postoperatively.

tissue. ${ }^{6}$ According to Hasselblatt et al, meningiomas with $>50 \%$ vascular components can be diagnosed as AMs. ${ }^{1}$ $\mathrm{AM}$ is rare; the 23 cases reported here accounted for $4.5 \%$ of all meningiomas treated surgically in our department during the same period. Although this incidence is slightly higher than reported in the literature, it remains low.

AM has features in common with other types of meningioma, but overall has a distinct presentation. Most meningiomas occur in middle and older ages; the mean age of patients in this series was 54 years, similar to meningiomas in general. In contrast to the typical female predominance of meningioma, the male/female ratio of AM cases in our series was 1.3 , indicating slight male predominance, which is consistent with other reports. For example, it was 1.37 in the series of Hasselblatt et al. ${ }^{1}$ Liu et al reported a male/female ratio of 1.08 for $27 \mathrm{AM}$ cases. ${ }^{3}$ Yet, in a report of the largest series of 93 AMs, Hua et al reported a male/female ratio of $0.94 .^{2}$ For spinal cord AMs, the male/female ratio was $1,{ }^{7}$ indicating no 


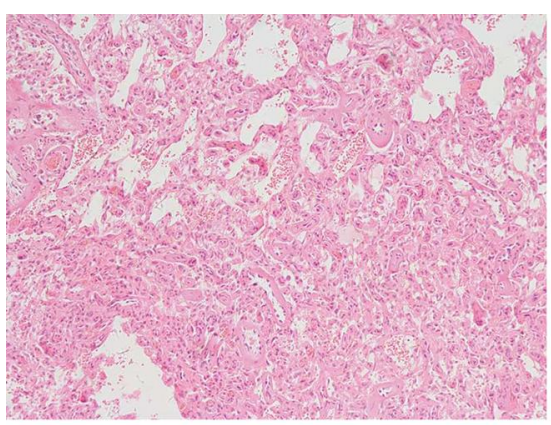

A

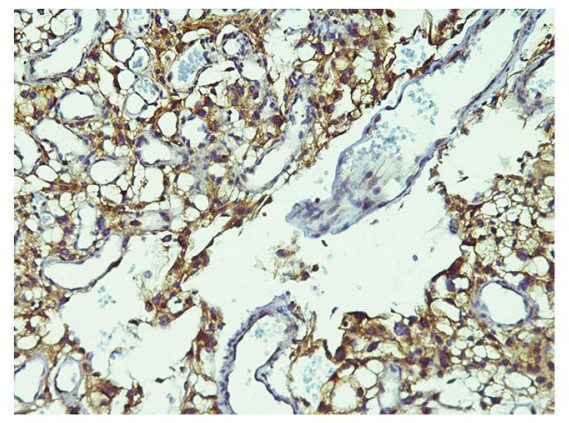

D

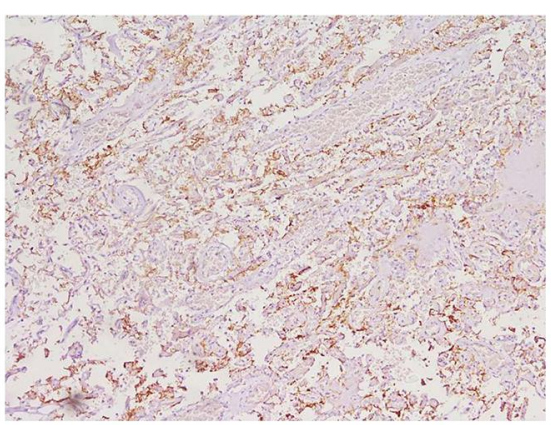

B

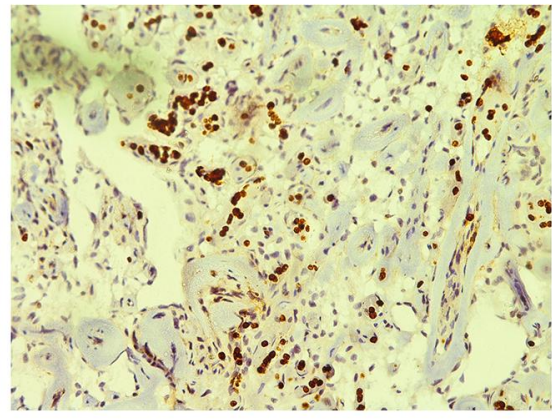

E

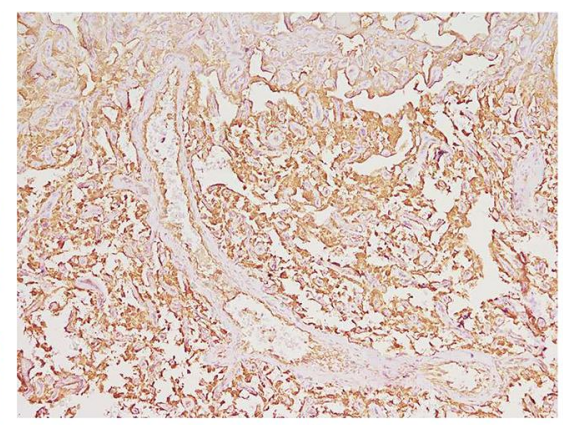

C

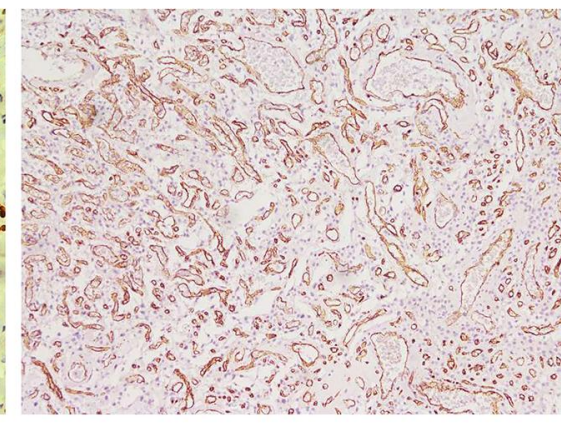

F

Figure 2 Representative pathohistological images. (A) Hematoxylin and eosin staining showed numerous blood vessels with intervening tumor cells. Immunohistochemical analysis showed strong EMA positivity of tumor cells (B), strong vimentin positivity of tumor cells (C), strong VEGF positivity of tumor cells (D), strong MMP9 positivity of tumor cells (E), and numerous vascular walls displaying CD34 positivity (F).

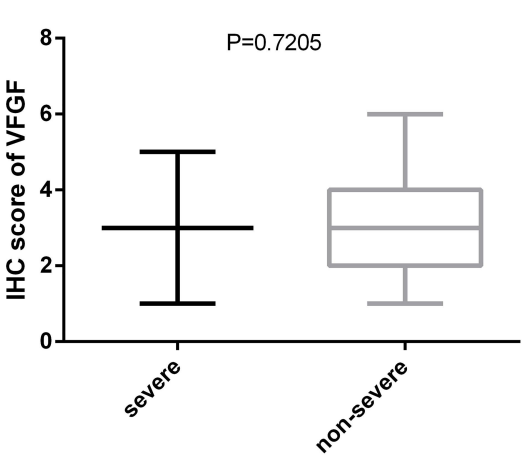

A

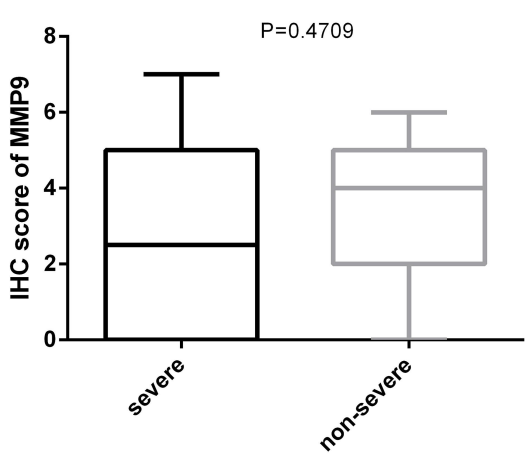

B

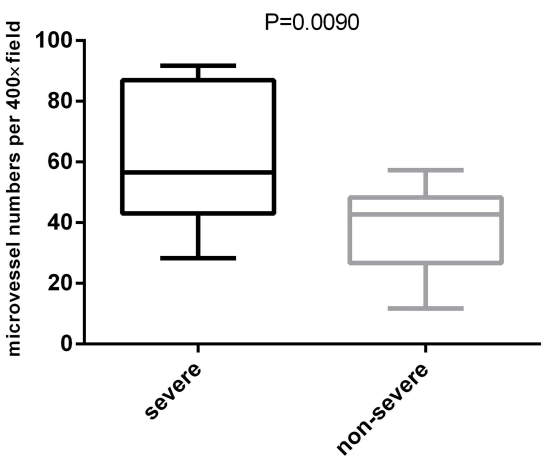

C

Figure 3 Boxes and whiskers graph show that among the IHC score of VEGF (A) and MMP9 (B) and MVD marked with CD34 (C), only MVD marked with CD34 was significantly related to the extent of PTBE $(\mathrm{P}<0.01)$ (GraphPad Prism 6.0).

apparent sex predominance. In addition, the predilection sites for AMs differ from those of common meningiomas; intracranial AMs occur most commonly in the cerebral convexity or parasagittal/falx region, as in $86.96 \%$ of our cases, and rarely at the skull base. ${ }^{1-3,8}$ In addition, on preoperative radiological examination, AMs show more obvious enhancement and vascular signs and less common meningeal tail sign than other meningiomas, which can make radiological distinction of AM from hemangiopericytoma (HPC), malignant meningioma, or hemangioblastoma difficult. Some cystic AMs can be also confused with metastases or gliomas before surgery. Our review indicates that AMs are generally hypo-intense on T1-weighted sequences (16/23) and hyper-intense on T2weighted sequences (18/23), while HPC is generally isointense on both T1-weighted and T2-weighted sequences. Besides, some researchers have suggested that the MRI ADC value differs between HPCs and AMs, but the analysis of more cases is needed to confirm this distinction. ${ }^{9,10}$ Malignant meningiomas have a rich blood supply and are associated with obvious PTBE, but they often have more 
aggressive biological characteristics, higher Ki-67 indexes, and less clear tumor boundaries compared with AMs; MRI spectroscopy and perfusion may help detect AMs from other malignant masses. ${ }^{11,12}$ Hemangioblastomas also have a rich blood supply, but mostly occur below the tentorium and cystic changes are commonly observed. ${ }^{11}$

PTBE is another important characteristic of AMs, different from other types of benign meningiomas. Our review demonstrates that $86.96 \%$ of AMs have PTBE, which is much more than $56.5 \%$ of common meningiomas. ${ }^{13}$ Possible factors related to PTBE in meningioma have been reported in a variety of studies, and include location, tumor size, vascular density, tumor-related blood obstruction, and histological subtypes, although the exact mechanism is still not well known. Some reports revealed that VEGF and MMP9 may be associated with the PTBE in meningiomas. ${ }^{14}$ Other reports also demonstrated that the PTBE in AMs may be associated with the abnormalities in VEGF or VEGF pathway. $^{15}$ Therefore, we analyzed whether VEGF, MMP9, or vascular density are related to the PTBE in AMs. Although our report revealed that only MVD marked with CD34 was related to PTBE in AMs, we cannot exclude the involvement of VEGF pathway in the occurrence of PTBE in AMs. Similar evidence was shown by Nassehi et al, who investigated the VEGF-A pathway in the development of PTBE in meningiomas. They found that capillary length and VEGFR-2 protein expression were higher in AMs with a higher edema index than in nonAMs, though VEGF-A protein did not differ significantly between them. ${ }^{15}$ All in all, our report indicated that angiogenesis may be involved in the PTBE of AMs, yet further molecular biology research is needed to reveal the mechanisms of PTBE in AMs.

Surgery remains the main treatment for most AMs, but the tumors' rich blood supply often makes the resection difficult. Poor surgical field visibility due to massive intraoperative bleeding may increase the possibility of cerebral and neural damage. ${ }^{1-3,8}$ In the 23 cases reported here, abundant tumor blood supply was observed during the surgical resection. Our experience indicates that AM removal should begin at the base to cut off the blood supply, followed by en bloc resection to the greatest extent possible. The three cases in this series that required blood transfusion were huge parafalx or skull base tumors, whose blood supply could not be cut off at the beginning of surgery. Recently, good results have been achieved when we performed the preoperative embolization to reduce tumor blood supply, especially for those in whom DSA showed a definite arterial feeder. Hence, better preoperative predictive ability is required for these tumors. For AMs that are likely impossible to be resected en bloc, preoperative embolization is useful and larger blood supply/autologous blood transfusion equipment should be prepared before surgery.

AM is generally associated with a good prognosis. Similar to the previous series from the literature, the patients in our series had no recurrence and survived in good condition through the follow-up period. ${ }^{1-3,8}$ This good outcome may also be attributable to the high rate of Simpson grade I resection and relatively short follow-up time. In cases of incomplete tumor removal, gamma knife, while not conventional radiotherapy, was recommended for a small residual tumor because of the non-invasive feature of AMs. One patient in our series underwent gamma knife therapy 3 months after the initial surgery due to the presence of a residual tumor in the cavernous sinus; no recurrence was detected during follow-up.

\section{Conclusions}

AM is a type of meningioma with a rich blood supply and distinct clinical and pathological features. It demonstrates a slight male predominance and is commonly localized at the cerebral convexity or parasagittal/falx region. Pathohistologically, it has benign biological characteristics despite frequent and severe PTBE. The extent of PTBE is related to MVD of tumors. Simpson I resection is the best treatment, and the prognosis is good after total tumor removal, while gamma knife is recommended for small residual tumor.

\section{Abbreviations}

AM, angiomatous meningioma; CT, computed tomography; CTA, CT angiography; DSA, digital subtraction angiography; IHC, immunohistochemistry; MRA, magnetic resonance angiography; MRI, magnetic resonance imaging; MRV, magnetic resonance venogram; MVD, microvessel density; PTBE, peritumoral brain edema.

\section{Ethical Approval and Informed Consent}

The Ethics Committee of The Huashan Hospital North of Fudan University approved the study protocol, which was performed in compliance with the Helsinki Declaration. Written informed consent was obtained from all of the patients. 


\section{Funding}

No funding was received for this research.

\section{Disclosure}

All authors declare no conflicts of interest.

\section{References}

1. Hasselblatt M, Nolte KW, Paulus W. Angiomatous meningioma: a clinicopathologic study of 38 cases. Am J Surg Pathol. 2004;28 (3):390-393. doi:10.1097/00000478-200403000-00013

2. Hua L, Luan S, Zhu H, et al. Angiomatous meningiomas have a very benign outcome despite frequent peritumoral edema at onset. World Neurosurg. 2017;108:465-473. doi:10.1016/j.wneu.2017.08.096

3. Liu Z, Wang C, Wang H, Wang Y, Li J, Liu Y. Clinical characteristics and treatment of angiomatous meningiomas: a report of 27 cases. Int J Clin Exp Pathol. 2013;6(4):695-702.

4. Simpson D. The recurrence of intracranial meningiomas after surgical treatment. J Neurol Neruosurg Psychiatry. 1957;20:22-39. doi:10.1136/jnnp.20.1.22

5. Weidner N, Folkman J, Pozza F, et al. Tumor angiogenesis: a new significant and independent prognostic indicator in early-stage breast carcinoma. J Natl Cancer Inst. 1992;84(24):1857-1887. doi:10.1093/ jnci/84.24.1875

6. Perry A, Rushing EJ, Louis DN, et al. Meningioma. In: Louis DN, Ohgaki H, Wiestier OD, et al., editors. WHO Classification of Tumors of the Central Nervous System. Lyon: International Agency for Research on Cancer; 2016:238.

7. Wu L, Yang T, Yang C, Deng X, Fang J, Xu Y. Surgical treatment of intraspinal angiomatous meningiomas from a single center. Neural Med Chir (Tokyo). 2015;55:328-335. doi:10.2176/nmc.oa.2014-0274
8. Nsir AB, Chabaane M, Krifa H, Jeme H, Hattab N. Intracranial angiomatous meningiomas: a 15-year, multicenter study. Clin Neurol Neurosurg. 2016;149:111-117. doi:10.1016/j.clineuro.2016.08.003

9. Liu L, Yin B, Geng D, Li Y, Zhang B, Peng W. Comparison of ADC values of intracranial hemangiopericytomas and angiomatous and anaplastic meningiomas. $J$ Neuroradiol. 2014;41:188-194. doi:10.1016/j.neurad.2013.07.002

10. Meng Y, Chaohu W, Yi L, Jun P, Songtao S. Preoperative radiologic characters to predict hemangiopericytoma from angiomatous meningioma. Clin Neurol Neurosurg. 2015;138:78-82. doi:10.1016/ j.clineuro.2015.08.005

11. Deb P, Sahni H, Bhatoe HS. Cystic angiomatous meningioma in the cerebellopontine angle mimicking hemangioblastoma. J Cancer Res Ther. 2010;6(4):560-563. doi:10.4103/0973-1482.77074

12. Uzunoglu I, Kizmazoglu C, Rezanko TA, Yuceer N. Intraparenchymal supratentorial papillary meningioma: a case report. Br J Neurosurg. 2018. doi:10.1080/02688697.2018.1454887

13. Iwado E, Ichikawa T, Kosaka H, et al. Role of VEGF and matrix metalloproteinase-9 in peritumoral brain edema associated with supratentorial benign meningiomas. Neuropathology. 2012;32:638-646. doi:10.1111/j.1440-1789.2012.01312.x

14. Tamiya T, Ono Y, Matsumoto K, Ohmoyo T. Peritumoral brain edema in intracranial meningiomas: effects of radiological and histological factors. Neurosurgery. 2001;49(5):1046-1052. doi:10.1097/ 00006123-200111000-00003

15. Nassehi D, Sorensen LP, Dyrbye H, et al. Peritumoral brain edema in angiomatous supratentorial meningiomas: an investigation of the vascular endothelial growth factor A pathway. APMIS. 2013;121:1025-1036. doi:10.1111/apm.12052
International Journal of General Medicine

\section{Publish your work in this journal}

The International Journal of General Medicine is an international, peer-reviewed open-access journal that focuses on general and internal medicine, pathogenesis, epidemiology, diagnosis, monitoring and treatment protocols. The journal is characterized by the rapid reporting of reviews, original research and clinical studies

\section{Dovepress}

across all disease areas. The manuscript management system is completely online and includes a very quick and fair peer-review system, which is all easy to use. Visit http://www.dovepress.com/ testimonials.php to read real quotes from published authors. 